EOMmun Communication et organisation

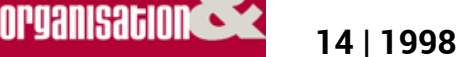

Les formations à la communication

\title{
Les NTIC et l'afrique : communication et utopie
}

Hélène Dufau-Rossi

\section{OpenEdition}

Journals

Édition électronique

URL : http://journals.openedition.org/communicationorganisation/2142

DOI : 10.4000/communicationorganisation. 2142

ISSN : 1775-3546

\section{Éditeur}

Presses universitaires de Bordeaux

Édition imprimée

Date de publication : 1 novembre 1998

ISSN : 1168-5549

Référence électronique

Hélène Dufau-Rossi, «Les NTIC et l'afrique : communication et utopie », Communication et organisation [En ligne], 14 | 1998, mis en ligne le 26 mars 2012, consulté le 10 décembre 2020. URL : http:// journals.openedition.org/communicationorganisation/2142; DOI : https://doi.org/10.4000/ communicationorganisation. 2142

Ce document a été généré automatiquement le 10 décembre 2020.

(c) Presses universitaires de Bordeaux 


\title{
Les NTIC et l'afrique : communication et utopie
}

\author{
Hélène Dufau-Rossi
}

1 Nul n'échappe plus à l'empire des « Nouveaux médias », ces NTIC qui finissent par ne plus être si nouveaux, tout en se perfectionnant chaque jour, et qui étendent leurs ramifications à la planète entière; l'Afrique n'est pas épargnée par le mouvement. Pourquoi le serait-elle, d'ailleurs, à suivre la chronique des bienfaits annoncés ? De fait, quelques acteurs particulièrement concernés ${ }^{1}$ développent des discours alléchants sur ce qu'il faut en attendre: le développement des NTIC va bouleverser les situations existantes, et permettre d'accéder à des trésors culturels incommensurables, d'introduire plus de démocratie dans les sociétés, d'intégrer les économies africaines au marché mondial, de sauvegarder les cultures, d'instaurer le dialogue entre groupes sociaux, d'amplifier la recherche, etc. Ce continent dénué entre tous pourrait trouver enfin la solution à tous les maux qui l'accablent, depuis maintenant des décennies, si ce n'est plus.

De fait, globalement, l'Afrique demeure le continent de toutes les difficultés. Elle rassemble les pays les plus pauvres du globe, ceux qui disposent des revenus les plus limités, (revenus monétaires, eau disponible, énergie consommée, ressources alimentaires). C'est toujours le lieu de la plus forte croissance démographique, de la plus faible industrialisation, elle n'assure pas son autosuffisance alimentaire, ses sols se dégradent de manière accélérée, ses ressources naturelles sont pillées. Elle ne représente plus que quelques \% des échanges internationaux, croule sous la dette, et un certain nombre de maux persistants, parmi lesquels la dictature, les conflits dénommés « de basse intensité ", mais aussi des inégalités sociales criantes ne représentent que la partie émergée d'un iceberg difficile à cerner ${ }^{2}$. Un certain nombre d'améliorations se produisent dans certains secteurs, des réalisations positives viennent en quelque sorte « miter » ce tableau ${ }^{3}$, mais pour l'instant on ne voit pas clairement se dégager un changement global qui inciterait à plus d'optimisme.

3 C'est dans un tel contexte qu'un certain nombre d'acteurs (géants des télécommunications, organisations bilatérales, multilatérales, etc.) interviennent, depuis 
déjà quelques années, pour stimuler le démarrage et le développement des techniques de communication les plus avancées, notamment l'accès à Internet, la radiotéléphonie et les communications satellitaires (téléphonie, transmission de données, télévision).

4 Il est frappant de constater à quel point leur discours est similaire à celui qui a été développé autour de la télévision, puis de l'informatique, ou dans le domaine plus modeste, mais tout aussi symbolique, des systèmes documentaires d'IST. Il s'agit à chaque fois d'introduire et de déployer des techniques investies de la capacité de «faire réaliser un bond dans le xxi ${ }^{\mathrm{e}}$ siècle ", de mettre enfin un terme aux divers dysfonctionnements existants ${ }^{4}$, de remédier aux carences de tous ordres constatables dans la vie de tous les jours.

5 Aussi, UTT, Banque mondiale, grandes compagnies internationales de télécommunications ${ }^{5}$, relayées par des compagnies nationales dont on a exigé la privatisation, se mettent-ils en devoir de développer les NTIC. On exige des gouvernements qu'ils abandonnent toute prétention sur leurs organismes de télécommunications, en général très mal gérés et dysfonctionnels ${ }^{6}$; on introduit Internet sur les campus, on développe des cabines de radiotéléphonie, des boutiques de télécommunications, on lance des cybercafés.

Dans ce vaste mouvement de changement piloté de l'extérieur, seule l'Afrique du sud est susceptible de pouvoir s'opposer aux propositions d'équipement et de service (fort onéreuses au demeurant) des grands opérateurs euro-américains. Son association avec Malaysia Telecom crée un pôle afro-asiatique qui pourrait générer un nouveau rapport de forces local dans le domaine des télécommunications.

7 Si les spéculations et les analyses de tendances optimistes vont bon train, on a cependant pour le moment beaucoup plus de questions que de réponses sérieuses, car le mouvement de la recherche sur le sujet n'en est qu'à ses débuts 7 . Cependant, l'étude du développement des NTIC en Afrique n'est pas à considérer comme d'un intérêt marginal : là comme ailleurs, se trouvent à l'œuvre les acteurs et les enjeux qui agitent et restructurent actuellement le reste du monde.

8 Pour notre part, nous avons donc choisi de noter ici quelques réflexions à la lumière de l'approche développée par Philippe Breton sur la «nouvelle utopie ${ }^{8}$ que serait devenue la communication, érigée depuis un peu plus de cinquante ans, en puissance régulatrice et salvatrice, arme " contre le Diable, le désordre, l'entropie, contre tout ce qui s'interpose entre les hommes et rend leurs rapports opaques, contre ce qui [...] conduit inéluctablement nos sociétés au désastre et à la dissolution du lien social »". Du coup, l'ensemble des techniques développées dans le champ de la communication acquièrent une valeur particulière, celle d'un "recours universel » à tous les maux ${ }^{10}$. Elles concourent globalement à alimenter l'utopie, mais aussi à développer ses "effets pervers ». La communication apparait, à l'analyse, comme le " $3^{\mathrm{e}}$ souffle du libéralisme " ${ }^{11}$, tout en prétendant en réparer les effets les plus visiblement délétères.

9 Cette analyse de l'utopie, et de sa mise en œuvre quasi totalitaire, nous paraît particulièrement pertinente. Malgré ses promesses, en effet, le désordre et la dissolution se répandent, sous des formes qui sont probablement liées, précisément, au déploiement massif des techniques de communication et d'information : perte du sens ${ }^{12}$ et des repères, développement exacerbé d'une concurrence économique mondiale féroce, concentrations d'entreprises, montée du chômage et aggravation de la misère jusqu'au sein même des sociétés développées, massification culturelle et exacerbation des revendications 
identitaires, développement des mafias et de la criminalité sous des formes hautement barbares (pensons par exemple à l'esclavage des enfants, sous toutes ses formes, mais aussi aux différents trafics, aux pillages de ressources naturelles ou au terrorisme), etc..

10 En outre notre expérience, pour modeste qu'elle soit, nous a sensibilisé aux réelles difficultés culturelles, ergonomiques et fonctionnelles que l'on rencontre lorsqu'il s'agit de développer des techniques et des pratiques orgarnisationnelles liées à l'information et à la communication en Afrique sub-saharienne ${ }^{13}$. Ces domaines y affrontent (comme partout ailleurs, de fait) des mécanismes fondamentaux liés au domaine cognitif, qui mettent en jeu des dimensions linguistiques, des représentations et des valeurs profondément intégrées aux cultures. Elles subissent, en tant qu'outillage immatériel de représentation, d'interprétation et de manipulation du monde, des contraintes qui sont peut-être plus fortes encore que celles que rencontre l'innovation technique dans n'importe quel autre domaine. La croyance dans la possibilité de transposer à l'identique des pratiques qui sont, somme toute, la quintessence d'un fonctionnement socioéconomique donné, relève d'une démarche idéologique profondément ethnocentriste, sinon (y compris dans ses dimensions économiques et politiques) culturellement impérialiste. Le développement de techniques de gestion de l'information scientifique et technique, tout comme celui des techniques de communication suppose, comme le souligne Ph. Breton, une "immense résonance sociale $»^{14}$. Celle-ci peut-elle s'acquérir, si ce n'est (au moins) au prix de bouleversements très volontaristes, voire de déculturation ?

11 Notre interrogation est donc la suivante : en quoi le développement des NTIC tel qu'il est proposé en Afrique relève-t-il d'une approche teintée d'illusion, pour ne pas dire de tromperie. En effet, l'instantanéité, la rapidité, la maîtrise du temps et de l'espace, voire l'indépendance à l'égard des sources d'énergie ${ }^{15}$ sont, apparemment assurés par ces techniques. On les pare de nombreux pouvoirs, susceptibles d'entraîner des améliorations rapides et radicales dans les domaines de la politique, de l'économie, de la culture. Mais les potentialités d'un ensemble technique ne valent que par les capacités dont disposent les sociétés à l'intégrer, compte tenu des contraintes complexes qui prévalent en leur sein: synergies entre les capacités d'investissement, de maintenance, d'organisation, d'appropriation culturelle, de résistance politique, etc.

\section{Une prolifération relative}

12 Une des conditions sine qua non de l'épanouissement attendu, notamment par l'insertion dans le marché mondial, est de disposer de l'équipement requis. Il est vrai que l'on assiste en Afrique à une relative "prolifération » des équipements et connexions pour accéder aux nouvelles technologies. (Il faut cependant considérer les parties Nord et Sud du continent à part, tant elles se distinguent du reste). Cette prolifération est malgré tout à nuancer.

13 La situation de l'Afrique sub-saharienne en termes d'accès aux moyens de communication contemporains est catastrophique, on l'a vu (cf. note (4)) : pour les lignes téléphoniques, elle est équipée entre 25 et 260 fois moins que le reste du monde ${ }^{16}$, avec une prédominance du sous-équipement - voire une diminution - dans les pays les plus instables politiquement. Elle connaît cependant une augmentation rapide de ses installations et connexions diverses (plus forte qu'en Europe). Mais que veut dire un 5,7 \% d'augmentation quand on part de moins de 2 lignes pour 1000 habitants? 

les capitales, et les zones rurales, qui rassemblent plus de $70 \%$ de la population, sont donc particulièrement sous-équipées. Pour ne pas parler des taux de pannes et des délais de mise en service.

C'est dans le domaine du «sans-fil » que les changements les plus rapides et les plus spectaculaires risquent d'avoir lieu. La téléphonie cellulaire prend son essor, au Sénégal, en Côte d'Ivoire, en Afrique du Sud et également dans les zones les plus déstructurées (comme la Guinée et le Zaïre). Les liaisons satellitaires augmentent rapidement. Les zones rurales peuvent être desservies par des cabines autonomes en énergie, et en connexions filaires.

16 Le nombre de paraboles de télévision satellitaire s'envole, passant de 3500 en 1989 (au Cameroun, au Gabon, au Sénégal, en Côte d'Ivoire), jusqu'à 120000 en 1994 (17) ${ }^{17}$.

Quant aux accès au réseau mondial Internet, elles sont vigoureusement appuyées par des institutions internationales diverses, comme l'US-Aid (Projet Leland, qui installe des lignes à $128 \mathrm{~kb} / \mathrm{s}$ ), ou l'Aupelf-Uref, qui donne des accès par son système de points Syfed sur le REFER (Réseau Francophone de la Recherche), après qu'ils aient été initiés, entre autre, par l'Orstom notamment, avec le réseau Rio, ou par des ONG (Healthnet, GreenNet, etc.)- La connectivité augmente tous les jours ( $c f$. le site de Mike Jensen) et la plupart des pays disposent actuellement (mai 98) de la possibilité d'accéder à l'ensemble des fonctionnalités d'Internet. Le courrier électronique est de loin le service le plus accessible et le plus utilisé.

18 En outre, des grands projets sont lancés pour augmenter les capacités de liaison du continent avec le reste du monde : par exemple Africa One (projet Banque mondiale, avec câblage en fibre optique), concurrencé par l'Afrique du Sud avec SAFE (Souther Africa Far East Cable System) et SAT-3 /WASC.

19 Il convient cependant de rester méfiant face à des données qu'il faut se garder de considérer dans l'absolu. Que signifient en effet des constatations comme «multiplication par deux ou trois... ou dix» des connexions, lorsque le chiffre de départ était... peu différent de zéro?

Que signifie également le fait que les pays soient désormais tous connectés si la connexion est de $9600 \mathrm{~b}$, ou même de $128 \mathrm{~kb}$, alors que le moindre citoyen connecté, dans les pays développés, à des prestataires de services, a accès à des débits de l'ordre de plusieurs dizaines voire centaines de Mo?

21 Peut-on prendre la mesure réelle de l'événement autrement que par référence avec les données correspondantes dans les pays développés? Mais les usages y sont-ils comparables? Et ne rendent-ils pas l'interprétation complètement différente ? Ainsi, on doit rester conscient que malgré une réelle augmentation du parc des ordinateurs, l'Afrique ne reste comptable que d'un pourcentage minuscule des équipements mondiaux : moins de $1 \%$ du parc mondial ${ }^{18}$. Que malgré le développement de sites et le succès du courrier électronique, ce n'est qu'une fraction minime de la population totale qui accède à ces services. Mais qu'un seul branchement peut induire des dizaines d'accès réels. 


\section{Utopie économique, économie de l'utopie}

\section{exporter du continent peut-elle vraiment changer rapidement? Les spéculations} agricoles qu'il peut chercher à commercialiser rencontrent à la fois une rude concurrence, et une consommation internationale stabilisée (c'est le cas du caoutchouc, du café, du cacao). Il serait intéressant d'observer des marchés spécifiques, comme celui des fleurs par exemple, d'y analyser les jeux de logistique et de systèmes de communication et d'information, et de mesurer l'apport des NITC. Comment peuventelles contribuer à développer une production industrielle exportable ? En ce qui concerne les matières premières minérales, les systèmes de prédations des richesses naturelles seront-ils combattus ou renforcés par l'emploi des techniques de communication? L'ensemble des échanges est conditionnée par les capacités d'organisation et de logistique. Comment les techniques de communication vont-elles pouvoir améliorer ces dimensions?

Les économies africaines sont connues pour être mal contrôlées, le secteur dénommé « informel» recouvrant une fraction majoritaire de l'activité économique. Ce sont les capacités de l'État à développer son emprise sur les personnes et le territoire qui auraient permis le développement de la modernité ${ }^{20}$. Les déficits de fonctionnement de l'État africain, depuis longtemps pronostiqué en voie de «dépérissement» peuvent-ils vraiment être comblés, et si oui, contribuer à un développement économique qui sorte de la prédation des richesses produites, telle qu'elle a eu lieu jusqu'à maintenant ? ${ }^{21}$. Le reste $\mathrm{du}$ circuit commercial demeure globalement mal organisé: voies de circulation insuffisantes, «taxes» occultes sur les transports, faiblesse des infrastructures. Quelles améliorations de fonctionnement seront-elles rendues possibles par les NTIC ? À quelles conditions?

28 Par ailleurs, l'innovation est devenue le moteur principal du fonctionnement du système économique mondial. Dans ce domaine, l'Afrique est frappée de handicaps 
particulièrement importants, dus notamment à la faiblesse de ses capacités de recherche/ développement, et aux difficultés de l'interfaçage entre les secteurs productif et financier ${ }^{22}$, dont la synergie conditionne la capacité de participer au "concert des nations", l'intégration au marché mondial. La recherche, est l'un des premiers secteurs visés, dans les pays francophones, par les organisations intervenant dans les domaines des NTIC. Dans quelle mesure, à travers quelles pratiques, pourra-t-elle vraiment bénéficier de ces techniques? Comment s'améliorera l'interfaçage avec le système productif ?

Une des caractéristiques persistantes des sociétés africaines demeure la faiblesse du pouvoir d'achat d'une fraction très majoritaire des populations, qui ne peuvent quasiment pas assurer les dépenses strictement nécessaires? Quels cyber-marchés peuvent-elles représenter? Il serait intéressant, alors qu'on souligne généralement le faible coût de ces techniques, notamment en termes de fonctionnement, de travailler à préciser ce que ces coûts représentent relativement aux ressources réelles de leurs supposés utilisateurs. Il en va de même pour les coûts d'équipement et de maintenance, lesquels représentent parfois plusieurs mois de salaires pour un fonctionnaire moyen. Il est vrai qu'une petite fraction de ces populations appartient à la jet society, va se faire soigner en Europe ou aux États-Unis, a une consommation luxueuse, et un niveau de vie outrageusement différent de ses concitoyens. Ne seront-ils pas, avec quelques grandes multinationales, et avec les organisations intergouvernementales supposées «aider " l'Afrique, les seuls bénéficiaires du développement des NTIC? Ne deviennent-ils pas comme « des expatriés » dans leur propre pays ?

D'autre part, on constate un développement important des activités mafieuses et des trafics divers ${ }^{23}$, une "criminalisation des échanges »; elles profiteront sans doute plus que toute autre activité des possibilités offertes par la téléphonie par satellite, le solaire, l'accès aux réseaux internationaux et le cryptage, et la perte de contrôle des États. Car il est facile de se laisser séduire par les possibilités de gains importantes, en complet contraste avec les opportunités qu'offrent les activités économiques licites.

31 En dernier lieu, on peut constater que les stratégies des grandes entreprises de télécommunications consistent à occuper le terrain, et à dépouiller les États de toute emprise sur le secteur des télécommunications, pour quand sera venu le moment d'engranger les bénéfices. Et ce avec le risque de voir disparaître toute possibilité d'orienter réellement les politiques dans ce domaine ; quel État pourra imposer la prise en compte des publics les plus démunis, si triomphe une logique d'investissement privé et de libéralisation totale du secteur des télécommunications? Quels bénéfices pourront-ils tirer de l'implantation de techniques qui leur échappent complètement, pour la conception, la maintenance et l'exploitation? Et finalement, quel endettement supplémentaire va finalement accompagner ces équipements, plus ou moins imposés par des partenaires extérieurs ? Ce sont là aussi des champs de recherche à défricher.

Certaines informations font d'ailleurs état du fait que certains États sont revenus sur la perte de contrôle qu'ils avaient consentis sur ce secteur. Seule l'Afrique du sud parait pour le moment capable de mettre en œuvre une stratégie d'échappement par rapport à l'offre européo-américaine d'équipement de télécommunications, dont elle conteste les coûts abusifs ${ }^{24}$. Elle propose une solution alternative, en coopération avec Malaysia Telecom, et l'on voit ainsi se dessiner un pôle Afrique du Sud/ Asie. On devra là aussi s'interroger sur la structure précise du capital, et la répartition des bénéfices entre les entreprises locales et les multinationales qui envahissent le marché africain des NTIC. 


\section{La dimension politique de l'utopie} consiste à affirmer qu'elles favorisent de nouvelles formes de démocratie. Elles ont parfois, en effet, pu servir la cause de communautés qui ont généralement du mal à se faire entendre (ONG, groupes militants, groupes de citoyens dans les situations tendues, comme en Chine ou en URSS, etc.) On les créditerait, notamment sous les aspects du Web, de la faculté de permettre des débats, des discussions, d'instaurer une certaine convivialité.

voudrions à ce propos soulever seulement deux points de réflexion: celui qui concerne la simple faisabilité de ces applications, et celui qui concerne les effets pervers de ces techniques.

Qu'il s'agisse de participation à des «forums civiques » évoqués par Pierre Chambat ${ }^{25}$, comme il en émerge aux États-Unis, et servant d'accompagnement aux politiques locales, ou de l'accès à des informations plus pertinente que celle qui est diffusée par les médias, il n'est pas certain que ces formes « hautement technicisées » de la démocratie soient une des priorités les plus urgentes des peuples africains. On ne perdra pas de vue la difficulté qu'il y aura, pendant encore longtemps, à faire dialoguer par écrit des citoyens analphabètes, non-équipés, dans des langues étrangères ou non-écrites souvent, et aux prises avec les dictatures que l'on sait. En outre, ces sociétés fortement « rencontrantes » (même si elles sont peu « communicantes » au sens de l'emploi de techniques complexes), risquent de ne pas trouver très crédibles des techniques distancées, alors qu'elles possèdent depuis longtemps des procédures locales de concertation et de consultation (même si ces formes ont été invalidées historiquement par l'intrusion de cultures étrangères).

$\mathrm{Au}$ contraire, il est à craindre que les hommes forts (anciens ou nouveaux), ne se trouvent être les seuls détenteurs - peut-être avec des multinationales, des commerçants ou des trafiquants puissants - de moyens de communication sophistiqués (radiotéléphones par exemple, systèmes de fichiers et d'écoutes divers) face à une population presque totalement démunie de moyens d'information... et d'organisation. On évoque ainsi la perte de contrôle des territoires par les États ${ }^{26}$, et plus encore, grâce aux nouvelles techniques de communication, le développement du pouvoir de "chefs de guerre », seuls équipés face à des populations démunies.

Car on oublie souvent trop facilement, à cet égard, que la maitrise de ces techniques assure aux pouvoirs qui en disposent une capacité quasi illimitée de surveillance et de contrôle. Pensons par exemple à l'espionnage total des autres pays du monde que pratiquent les pays du pacte Ukusa, à travers le réseau Échelon, grandement facilité par l'emploi des techniques de communication et de traitement de l'information ${ }^{27}$. Ces techniques peuvent servir des objectifs civils comme militaires, et exigent des équipements de haut niveau.

38 Il sera indispensable de procéder à des études fines et détaillées qui permettent d'observer leur insertion dans la vie politique africaine, afin de mesurer leurs effets réels. 


\section{Une utopie culturelle universelle}

39 L'introduction des NTIC concerne aussi le domaine culturel : la sauvegarde du patrimoine, la diffusion, et l'acquisition de savoirs, la formation. Dans ce domaine, l'utopie, c'est vouloir croire ou faire croire par exemple que le patrimoine culturel va être préservé, que la distance va être comblée entre les sources du savoir et les individus. Là aussi, nous pouvons porter quelques interrogations sur les effets, lesquelles ne sont d'ailleurs pas spécifiques à l'Afrique.

Car on ne peut oublier, en extrapolant les effets extraordinaires de ces techniques dans l'abolition du temps et de l'espace, que la distance peut aussi être culturelle. Et l'on retrouve là une dimension universelle de l'utopie : l'accès au support ne veut pas dire accès à la connaissance ${ }^{28}$. Le multimédia est à cet égard un exemple hautement significatif : on polarise généralement l'analyse sur l'attractivité du support, comme si celle-ci pouvait suppléer le manque d'intérêt, de motivation, de curiosité, et générer l'envie de savoir, qui relève, on le sait, d'une subtile alchimie socio-culturelle, mais aussi pédagogique. Les travaux menés sur le fonctionnement cérébral et les dimensions psycho-affectives, voire physiologiques des processus cognitifs démontrent que l'apprentissage nécessite des stratégies étroitement adaptées aux capacités et aux caractéristiques individuelles des apprenants, et bien plus qu'une simple mise en forme médiatique. La répétition, et une certaine forme d'effort, d'encadrement subtil et individualisé ne peuvent être exclus du processus. Au contraire, l'apprentissage suppose "de la tolérance à l'ennui et aux difficultés $»^{29}$ afin de franchir la barrière des 20 à 40 premières minutes de l'inconfort qu'est la confrontation à de la nouveauté, et d'atteindre le «second souffle» du mental, qui seul permet d'atteindre le stade de la "pensée formelle» décrit par Piaget ${ }^{30}$. Un système d'accès aux connaissances trop ludique empêche d'atteindre le stade de la pensée formelle. Avec le jeu des médias, la domination de l'aspect ludique, divertissant et facile ${ }^{31}$, semble au contraire générer une plus grande incapacité à fournir l'effort de lecture et d'information nécessaire, qu'on le veuille ou non, à l'acquisition d'une véritable culture. L'utopie, comme le souligne P. Breton, relève d'une confusion entre accès à l'information (nous dirions même aux données), et l'accès à la connaissance, et aboutit à «augmenter le fossé entre ceux qui sont dotés par la nature ou par l'environnement familial, [du désir de savoir], et ceux qui auront besoin d'un système éducatif attentionné pour le faire naître et l'entretenir. Or ces derniers sont et seront une large majorité et les premiers l'exception $»^{32}$.

41 Ainsi, le surinvestissement dans le multimédia ou le réseau, comme ressources d'apprentissage, constituent une approche trop simplificatrice du phénomène, et fonctionnent en plein dans le cadre de l'utopie de la communication. En Afrique, le risque peut venir du fait qu'en accord avec une vision utopique de ces médias, et la prédominance de solutions conçues ailleurs, le développement (tout relatif) des NTIC induise un désinvestissement des systèmes d'éducation habituels, au demeurant coûteux et insuffisants, et bloque la recherche de solutions originales, au profit de techniques qui ne peuvent tenir leurs promesses.

Dans des pays où une grande majorité de la population est rurale et ne maitrise pas les grandes langues occidentales, vecteurs principaux des contenus portés par les NTIC, la question des langues africaines ne sera toujours pas résolue : très nombreuses, les langues africaines ne sont pas écrites pour la plupart, sauf quelques langues «de grande 
extension $»^{33}$, et n'ont donné lieu à des productions écrites que quelques langues seulement. Or on n'oubliera pas que les NTIC supposent, d'une façon ou d'une autre, une bonne maitrise de la lecture, pour ne pas dire de l'anglais, ou du français. Et la francophonie, par exemple, n'est pas, quoi que l'on dise, une réalité généralisée ; seuls quelques \% de la population maitrise vraiment le français, ce qui contribue à la problématique de l'extraversion culturelle. En outre, on connaît l'importance, pour accéder au savoir, de l'usage des langues maternelles. Le dilemme reste entier.

Par ailleurs, on ne peut affirmer que la connaissance spécifique, générée par l'Afrique au cours des siècles, et qui a fait la preuve de son efficacité dans de nombreux domaines, sera respectée, relayée et mise en œuvre à travers les NTIC. Il en va de même de ses dimensions artistiques et culturelles (chanson, théâtre, littérature). Ce que Bill Gates n'a pas choisi d'acheter et de mettre en scène ne perdra-t-il pas toute disponibilité ? En outre, le changement de statut qui transforme des objets rituels, instruments l'organisation symbolique de l'univers, en objet d'art à consommer, est-il compatible avec un véritable respect des cultures ? Et comment peut-on être rassuré sur la sauvegarde des concepts et mécanismes de base des sociétés africaines (définitions des relations entre individus, au sein de la famille, entre groupes, par genres, avec l'univers ambiant, par exemple), si leur transmission est prise en charge dans des langues qui ne peuvent en rendre véritablement les nuances, ou par des acteurs autres que ceux qui connaissent ces réalités (notamment des auteurs et éditeurs occidentaux) ? À moins qu'il ne s'agisse précisément de faire disparaître les systèmes de valeurs de ces sociétés? L'acquisition et la conservation de données allant dans le sens des intérêts des apprenants, individuellement et globalement, exige des adaptations trop spécifiques pour pouvoir être le fait de produits de masse standardisés... Elles ne pourront être le fait que de démarches entreprises par les pays africains eux-mêmes, nécessitant des prises de position politiques fermes, et une appropriation de moyens conséquente.

Est-il vraiment nécessaire et efficace de mettre en œuvre des équipements fragiles et compliqués pour transmettre le fonds culturel vivant des sociétés? Fort heureusement, ces traditions et connaissances continueront à être transmis à peu de frais et selon les procédures habituelles de la structuration sociale (initiation, transmission entre générations, au sein des groupes "de genre", etc.). Mais alors, les techniques sophistiquées dont il est question concerneront principalement des savoirs d'autres origines, et a vu les limites qui sont les leurs.

Un domaine spécifique constitue un des grands espoirs de l'application des NTIC dans un domaine particulier du savoir : celui de la recherche, qui constitue d'ailleurs le principal des interventions d'assistance de la France ou des instances de la francophonie sur le continent: équipement des universités, accès à des serveurs, mise à disposition du courrier électronique, etc. Néanmoins, si on se rapporte aux mécanismes mis en évidence par B. Latour ${ }^{34}$, la recherche scientifique n'est pas une activité existant pour elle-même, qui doive générer de façon autonome une évolution dynamique des sociétés. Bien au contraire, elle ne peut fonctionner que grâce à son intégration dans un tissu complexe d'acteurs qui participent à sa progression, et jouent avec elle un jeu de synergies réciproques, qu'il s'agisse d'industriels, de politiques, de consommateurs, devenant partie prenante de ses objectifs. Et à cet égard la recherche africaine se trouve plutôt, de par ses origines et ses moyens, comme une pièce rapportée artificiellement dans un milieu qui est loin de lui offrir les conditions de son épanouissement. Ni la formation de ses chercheurs, ni leurs conditions de travail, tant sociales qu'organisationnelles, ni ses 
ressources, souvent exogènes et aléatoires, ni les acteurs économiques parmi lesquels elle est censée trouver des partenaires ne lui permettent un véritable développement ${ }^{35}$. On retrouve ici les interrogations que nous avons exposées concernant le secteur économique.

D n'est, bien entendu, pas question de dire que le développement des NTIC en Afrique n'est pas possible, ou ne sera d'absolument aucune utilité. Sans doute certaine secteurs marchands, administratifs, éducatifs, scientifiques, sociaux, pourront-ils être bénéficiaires d'une mise en œuvre élargie. Encore reste-t-il à en mesurer le coût véritable, en relation avec les capacités locales de financement, sinon d'endettement, avec les conditions et processus d'appropriation, et les pratiques réelles. On peut probablement prédire un bon avenir au téléphone cellulaire, à la radio (notamment dans les langues nationales, comme c'est déjà le cas), au fax, voire au courrier électronique, éventuellement relayé par porteur spécial dans la phase finale de délivrance du message. En fait, chaque fois que le médium respectera le message, sa langue, l'échange entre les acteurs, ou que l'appropriation de l'outil restera conforme au système de valeurs. Car en Afrique, il n'y pas encore tout à fait de société de masse, et il existe encore une valorisation importante des relations individuelles, nécessaires à la cohésion sociale.

Cependant, à l'examen, l'utopie de la communication, sous les espèces de techniques à son service, se manifeste de façon criante en Afrique, dans la mesure où la réalité qu'elle prétend améliorer est très éloignée des promesses qu'elle fait. Les outils d'information et de communication ne risquent-ils pas d'apporter, essentiellement, de l'eau aux plus grands moulins, des ressources aux mieux dotés, des forces aux plus puissants, des capacités de nuisance aux moins scrupuleux ? Il y malheureusement, de notre point de vue, beaucoup de chance pour que seule une petite minorité, celle des acteurs les plus puissants, les mieux dotés en argent, en savoirs occidentalisés, en armement, les mieux intégrés à des réseaux larges et internationaux - fussent-ils mafieux - soient les principaux bénéficiaires de cette évolution. Sans doute quelques réseaux commerçants réussiront-ils à renforcer leur capacité d'intervention, quelques associations citoyennes et démocratiques à mieux faire entendre leur voix, quelques ONG à améliorer leur capacité d'assistance, quelques organisations internationales à accélérer leurs activités. Mais la capacité de prédation et de domination de quelques uns ne risque-t-elle pas de sortir renforcée par l'usage d'armes aussi phénoménales qu'inaccessibles à la masse de la population? On peut finalement se demander si le montage de cette utopie ne joue pas, également, un rôle essentiel de camouflage idéologique qui cherche à faire oublier les effets pervers de ces techniques? Qui cherche à faire oublier combien, contrairement à ses affirmations, ses capacités de destruction sont importantes : mondialisation effrénée de l'économie, avec son cortège de drames humains, effets démultiplicateurs sur les capacités terroristes et criminelles, effets de contrôle sur les individus, effets de fragmentation des sociétés, développement de l'individualisme et perte de contact réel avec l'autre. 


\section{NOTES}

1. Et pas nécessairement désintéressés. Ce sont soit des organisations internationales intervenant depuis longtemps dans le «développement africain », comme la Banque mondiale, l'US-Aid, des organismes culturels ou de recherche, comme l'ACCT, mais aussi l'ORSTOM, des organismes spécialises comme l'UIT, etc.; ou des entreprises multinationales de communication et télécommunications, américaines, européennes, voire malaise. Elles développent des projets et des discours mirifiques sur les promesses des NTIC pour l'amélioration des différents secteurs de la vie en Afrique (éducation, santé, vie politique, commerce, gestion des ressources naturelles, des villes, etc.).

2. Pour plus de détails, on se rapportera aux analyses récurrentes de la Banque mondiale sur la situation africaine.

3. Il est difficile de faire un bilan global de ce qui "va mieux » sur le continent; on pourrait en prendre pour exemple le secteur de l'exportation de fleurs coupées, pour lequel l'Afrique devient un concurrent de plus en plus sérieux sur le marché international. Il est délicat d'évaluer l'effet d'entraînement d'un tel progrès sur une situation qui comporte beaucoup d'urgences cruciales (développement de la culture et du trafic de drogues, conflits, épidémies, dégradation de l'environnement, manque d'eau, etc.).

4. Avec ses $20 \%$ de la population mondiale, l'Afrique ne dispose pour le moment que de $2 \%$ des lignes téléphoniques, avec une densité globale très faible ; souvent moins de deux lignes pour 1 000 habitants en moyenne (contre 48 en Asie, 280 en Amérique, 314 en Europe - Est et Ouest - et 520 pour les pays à hauts revenus. C'est pourquoi elle se présente à la fois comme un immense marché potentiel, hautement convoité, mais aussi comme un « laboratoire " grandeur nature où tout peut se produire, avec l'introduction des techniques les plus performantes, sur les plans social, culturel, politique, économique

5. AT\&T (USA), Alcatel (France, Europe), Northern Telecom (Amérique Nord, Europe), Siemens (Allemagne, Europe, USA), Ericsson (Suède, Europe, USA), NEC (Japon), Motorola (USA, Europe), Malaysia Telecom (Malaisie)

6. Difficultés énormes de recouvrement des taxes téléphoniques, délais extrêmement importants d'accès aux raccordements -souvent plusieurs années -, taux de pannes les plus importants du monde.

7. L'évolution de cette situation a commencé à générer un certain nombre de recherches; on notera par exemple, sans prétention à l'exhaustivité, les institutions et personnes ressources suivantes (principalement sur la zone de la francophonie) pour leurs interventions et travaux :

- le Centre d'Etudes des Médias (CEM/Université de Bordeaux HT), qui se penche depuis longtemps sur les médias, notamment en Afrique (publication récente de l'ouvrage du Pr. A.-J. Tudesq, L'espoir et l'illusion: actions positives et effets pervers des médias en Afrique subsaharienne, MSHA, 1998, qui concerne essentiellement les médias (presse, radio, télévision)

- le Laboratoire REGARDS, UMR ORSTOM/CNRS, et notamment les recherches d'Annie ChéneauLoquay (); voir par exemple Annie Chéneau-Loquay • Les configurations socio-territoriales africaines sont-elles un atout pour l'insertion des NTIC ?. Netcom / Actes du colloque de Palma Géoespace et cyberespace vol. 12. juin 1998, 26 p. voir également le site Web " Africanti ", www / regards.cnr s-fr/africanti ; nous devons à Annie Chéneau-Loquay la plupart des informations sur les recherches en cours : 
- Pascal Renaud, informaticien de l'ORSTOM, «père » du réseau Rio, actuellement en poste a l'UNTTAR à Genève (); il a produit un CD-ROM « Internet au Sud », aux éditions de l'ORSTOM, qui rassemble des textes de réflexion, des cartes et des informations pratiques

- l'Institut Panos, doté de centres à Pans (10. rue du Mail, 75002 Paris), Londres et Washington, affiliés à Panos International, à Ottawa,

- le CRDI, avec son projet Acacia, notamment au Sénégal, associé a

- l'Observatoire interafricain des télécommunications de Dakar, au sein duquel Gaston Zongo (http : / /www.esmt an) s'est penché sur les télécentres sénégalais; le suivi des équipements y est complété par un nouveau regard sur leurs dimensions sociales

- le suivi de Mike Jenaen, qui marient un site sur la connectivité, à l'adresse : http : //www.org/ africa/afstat.htm

- le Centre for International Development and Conflict Management à l'Université du Maryland at College Park, •etc

- un premier ensemble de recherches publié dans le $n^{\circ} 111$ de la Revue Tiers-Monde, dirigé par Y. Mignot-Lefebvre, ainsi que ses travaux ultérieurs

Par ailleurs, l'Afrique du sud vient de créer un Centre for Global Access to Information and Communication Technologies, destiné a favoriser un meilleur accès possible aux NTIC, une initiative du gouvernement, assisté du CRDI.

8. Philippe Breton, L'utopie de la communication: le mythe du village planétaire. Pans: la découverte, 1997, 171 p. (Poche Essais)

9. Ibid., p. 11

10. Ibid., p. 125

11. Ibid., p. 126

12. Zaki Laïdi, Un monde privé de sens. Pans : Fayard, 1994, 333 p.

13. En tant qu'expert des Nations-Unies (Food and Agriculture Organization) pour le développement des systèmes d'information documentaire dans le domaine de l'agriculture et du développement, dans le cadre d'une dizaine de missions en Afrique de l'Ouest, et du Centre principalement Cela nous a permis d'identifier, au-delà d'une culturelle considérable un fonds très vivace de conceptions et de valeurs communs, qui se manifeste notamment dans les pratiques liées a la communication et à l'organisation de l'information. Cette expérience a suscité une thèse en Sciences de l'information et de la communication: Hélène Dufau-Rossi, La communication de l'information scientifique et technique en Afrique: des limites de la réplication du modèle occidental comme facteur de développement, thèse de doctorat, Université de Bordeaux III, 1996, 585 p.

14. P. Breton, Op. Cit., p. 17

15. Il existe actuellement des possibilités d'équiper de panneaux solaires des téléphones ou des ordinateurs, leur assurant ainsi une indépendance suffisante par rapport aux structures classiques de fourniture d'énergie, largement insuffisantes jusque là en Afrique. (Entretien avec A. Cheneau-Loquay, responsable d'un projet de recherche sur les NTIC en Afrique dans le cadre de l'UMR REGARDS.)

16. Les données chiffrées sur les équipements africains et leur interprétation proviennent pour la plupart de l'article d'Annie Chéneau-Loquay, «Quelle insertion de l'Afrique dans la société de l'information?» Les configurations socio-territoriales africaines sont-elles un atout pour l'insertion des nouvelles technologies de l'information et de la communication? », In : Pascal Renaud (Ed) Internet au Sud [CD-ROM], Orstom, 1998

17. Voir Abdoul Ba, Paraboles et démocratie, l'Harmattan,1995.

18. Lucas Patriat, "Télécommunications: les problèmes de base spécifiques à l'Afrique ", Marchés tropicaux, 19 déc. 1997, p. 2 780-81

19. Olivier Céna, "Les surfeurs de Dakar", numéro hors série de Télérama- "Au bonheur d'Internet », janvier 1998, p. 30-31; et enquête de Jean Marchai en mai 1997, citée par Annie 
Cheneau-Loquay dans "Quelle insertion de l'Afrique dans la «société de l'information »...». Op.cit. (cf. note 19).

20. Voir P. Matarasso, « Essais sur le développement, l'énergie, l'économie et le langage, PIRSEMCNRS, [inédit], 1991, $406 \mathrm{p}$.

21. Voir les travaux de J.-F Bayart et J.-F. Médard, par exemple J.-F. Bayart, L'État en Afrique : la politique du ventre. Paris : Fayard, 1989, 439 p.; J.-F. Médard, «L'État patrimonialisé ». Politique africaine, $n^{\circ} 39$, septembre 1990, p. 2536

22. Cf. analyse de B. Latour, La Science en action, La Découverte, 1989.

23. Voir par exemple les travaux d'Alain La brousse, notamment : «Production illicites et trafic des drogues ", p. 271-285 In : D. Bach, dir. Régionalisation, mondialisation et frangmentation en Afrique sub-saharienne, Paris : Karthala, 1998.

24. Voir «Une solution africaine a un problème africain ». Communication Africa/Afrique, aoûtseptembre 1997, p. 13 et 19.

25. Maître de Conférences à Paris X-Dauphine, chercheur en sciences politiques À l'IRIS (Institut de Recherche et d'information socio-économique); voir l'interview dans le numéro hors série de Télérama « Au bonheur d'Internet ", janvier 1998, p. 36-41.

26. Notamment les travaux de J. Igue, J. Egg, B. Badie et M.C. Smouts, Z. Laïdi, F. Constantin, sur les relations internationales, ainsi que ceux de D. Bach, notamment l'ouvrage récent: Régionalisation, mondialisa bon et fragmentation en Afrique subsaharienne, D. Bach, dir.. Pans : karthala 1998, 319 p.

27. «Attention, vous êtes sur écoutes » - Dossier, Courrier international, $n^{\circ}$ 387, 2-8 avril 1998, p. 39-41; voir aussi l'ouvrage de Fabrizio Calvi et Thierry Pfister, L'œil de Washington, Albin Michel, 363 p. Voir aussi le rapport de la Commission d'évaluation des choix technologiques et scientifiques de la Direction générale de la Recherche au Parlement européen, «Évaluation des techniques de contrôle politique ", 1998.

28. Cf la distinction entre « information » et « connaissance » soulignée par Denis de Rougement, «Information n'est pas savoir », Diogène, n 116, 1981 ; repris par Breton, Op. cit., p. 140

29. Hélène Trocmé-Fabre, J'apprends donc je suis: introduction à la neuropédagogie, Éd. d'organisation, 1987, p. 162.

30. Cité par Trocmé-Fabre, Op. cit., p. 163.

31. P Breton, Op cit., p. 142.

32. Ibid., p. 146

33. Voir les travaux de Mwatha Ngalasso, comme par exemple «Le dilemme des langues africaines ", Notre Librairie, $\mathrm{n}^{\circ} 98$, juillet-septembre 1989, p. 15-21 ; voir également «Les langues nationales : dossier», Le courrier ACP/CEE, nº 119, janv.-fév. 1990, p. 44-82.

34. Bruno Latour. Op. cit.

35. Hélène Dufau-Rossi, Op.cit.; voir aussi les travaux du département H. de l'ORSTOM, notamment J. Gaillard, Les chercheurs des pays en développement: origines, formations, pratiques de la recherche et production scientifique, elle éd., Paris : ORS-TOM, 1989, 220 p., J. Gaillard et R. Waast, "La recherche scientifique en Afrique ", Afrique contemporaine, $\mathrm{n}^{\circ} 148$, 1988, p. 3-2. B. La tour, dans La science en action, ne fait qu'évoquer la recherche africaine, pour en signaler l'insignifiance relative au plan international. Les principaux indicateurs de mesure de l'activité de recherche (brevets, publications, et notamment: dans les "grandes revues ", ne la créditaient en effet que de $0,38 \%$ de la recherche mondiale au milieu des années 80 . 


\section{RÉSUMÉS}

De nombreuses actions sont développées pour intégrer l'Afrique dans le monde des NTIC, lesquelles constitueraient la panacée à tous ses maux. Dans quelle mesure le discours sur les avantages attendus n'est-il pas un avatar de la vision utopique développée autour de la communication? La «flambée » constatée des équipements ne se traduit cependant en chiffres absolus que par des changements modestes. Essai de réflexion critique sur l'économique, le politique, le culturel. Pourront-ils réellement bénéficier de l'introduction de ces techniques?

Numerous interventions meant to insert Africa into the global NTIC world tend to present them as a panacea for all its difficulties. This approach is analyzed as a new manifestation of the communication Utopia. The recorded " phenomenal growth " of equipments finally amounts to little absolute quantities. Economy, politics and cultural fields are considered in a critical point of view : will they really benefit from this insertion?

\section{INDEX}

Mots-clés : NTIC, Afrique sub-saharienne, technique, développement social, développement économique, vie politique

\section{AUTEUR}

\section{HÉLÈNE DUFAU-ROSSI}

Hélène Dufau-Rossi est Maître de conférences en communication à l'IUT de Bordeaux 1. Elle a été plusieurs années spécialistes des systèmes d'information documentaires au CNRS, consultant auprès de la FAO, et dans le privé. Sa thèse de doctorat porte sur l'adaptabilité des systèmes d'IST en Afrique. Ses principaux champs de recherche sont la dimension Socio-économique de l'information scientifique et les Systèmes documentaires d'IST. 